\title{
Exchanging resilience for persistence: perspective for a new era in training
}

\author{
Hannah R. Turbeville \\ University of Mississippi Medical Center, Jackson, Mississippi, USA.
}

W hile we have all been guilty of overusing the word "unprecedented" this year, one buzzword I haven't heard as much as I expected is resilience. Resilience has become a mainstay of training as medical schools, residency programs, and research training programs alike seek to matriculate trainees that will be successful despite the trials inherent to these training paths. The identification of a resilient individual is a key obstacle to this goal. One definition of resilience is "the capacity to respond to stress in a healthy way such that goals are achieved at minimal psychological and physical cost." In other words, "resilient individuals 'bounce back' after challenges while also growing stronger" (1). I would like to propose that this definition promotes the misconception that achievement is worthwhile at the expense of any psychological or physical cost at all. Why is the ability to achieve arbitrary benchmarks, regardless of cost, used in the adjudication of fitness for admission to a chosen career field? An argument could be made that resilience speaks to the individual's ability to perform the job for which they are applying despite obstacles, setbacks, or personal trials. I suggest that the individual's commitment to pursuing that path identifies the same qualities and can easily be quantified using their resume, their educational history, and commonly asked interview questions.

Another misconception about resilience is that we often picture it as tremendous success in the face of difficulty. However, sometimes the simple decision to keep going, to wake up and try again, demonstrates the same spirit and determination. I suggest a suitable replacement for resilience: persistence. Persistence is a trainee who failed but chose to try again. It is a trainee with limited resources or support finding their way to a mentor's door. Persistence is progress despite the everyday complexity of our lives. Maybe it's time we began seeing the persistence that is already evident in the resumes of our trainees and assuming resilience rather than demanding proof or attempting to train it into them.

I am proud of the persistence that APSA has demonstrated this year, both in our accomplishments as an organization as well as the success of our individual trainees. APSA responded early to challenges imposed by the pandemic. When the 2020 Joint Meeting was cancelled, we were able to pivot quickly and provide a subset of our programming on a virtual platform. While several members of our executive team were defending dissertations and preparing for graduation, we created a virtual residency luncheon, a highlight of each year's meeting, for the very first time and in less than four weeks. When there was clearly no end in sight to infection control measures impacting trainee research opportunities, our Justice, Equity, Diversity, and Inclusion (JEDI) committee innovated a Virtual Summer Research Program (VSRP) from the ground up in under two weeks. In that short amount of time, they created a mentor-mentee matching system, a structured program outline, a journal club schedule, and most importantly - funding for many students. For those unable to be paired with mentors, many students were given the opportunity to attend a virtual course taught by Jackson Laboratories free of charge. Our Residents, Fellows, and Junior Faculty (RFJF) committee responded early by starting multiple survey-based research projects to gauge the pandemic's impact on all stages of the physician-scientist training pipeline, which was presented

Reference information: / Clin Invest. 2021;131(18):e150828. https://doi.org/10.1172/JCI150828. This article is adapted from a presentation at the 2021 AAP/ASCI/APSA Joint Meeting, April 10, 2021. Copyright: @ 2021, American Society for Clinical Investigation.

earlier this afternoon. These studies as well as data from the VSRP are approaching publication as we aim to share what we have learned this year with the larger scientific community. This year's committee reports are riddled with achievements that would be impressive in a typical year. This year, they are outstanding. Our Executive Council broadened APSA's reach and increased our impact with limited resources and time as they all continued to progress in their own training paths. As I mentioned, several of our members have defended their dissertations throughout the year and matriculated back to medical training. I am so proud to have been a part of this group and all that we have achieved. Our progress this year is truly a reflection of group effort and collaboration.

Today we're not only reflecting on our achievements - we're moving forward. This year we aim to maintain the momentum we have gained this year, with continuing initiatives from almost every committee. We are expanding and lengthening the VSRP with some exciting new surprises in store. Many of our research projects by the JEDI, RFJF, and policy committees are ongoing. While we are hopeful to move many events back to an in-person format, we have created a Virtual Content ad hoc committee in the hopes that we can maintain the opportunity to join virtually for those unable to travel for any reason. We are also using this opportunity to expand and improve our virtual webinar offerings. We have exemplified a spirit of iteration, improvement, and innovation that I expect to continue for years to come.

We have all demonstrated in the past year that we have more potential for resilience than we expect. I don't think many of us could have provided concrete examples to prove that we could continue to become doctors, researchers, parents, or caretakers in a worldwide pandemic. But we did. We persisted by performing our jobs from home or stretching that last paycheck from a lay- 
off. We persisted despite physical isolation. Many persisted by simply continuing to be true to themselves despite systemic prejudices against their identity. In the face of these and many other unprecedented challenges, we forged ahead. Over half a million people have perished from COVID-19 in addition to the untold morbidity and mortality of pandemic-impacted diseases and processes, such as exacerbation of depression, substance use disorders, or food scarcity. But still we persist. In recognition of the innumerable and unique challenges that each of us has been through, in realization of the fact that we will never truly know the multifaceted impact of this year on each individual, let us finally stop asking one another about resilience.

Our community's fascination with resilience says more about the systems we allow to perpetuate than about any single applicant pool. How well can an applicant navigate the hidden curriculum? How few resources can be provided for the applicant to be successful? Instead of searching for more resilient trainees, let us instead focus on changing the systems that require it This will not be easy; established systems resist change by nature. I have realized over the past few months that there are some notable differences between established organizations and newer ones. While established organizations have a demonstrated track record and have experienced the iteration that leads to success and longevity, there is a danger of becoming entrenched in the maintenance of tradition at the expense of innovation. Newer organizations have less institutional memory and less certainty of longevity, but this newness breeds a certain proclivity for innovation and risk-taking. The challenge for us as we continue to establish ourselves together as an organization and individually as physician-scientists is to establish our place and demonstrate our persistence while not losing our willingness to innovate and change. May we never forget what we learned during our training years, during this year especially, as we find new ways to move forward. This will not be easy, but no matter the challenge, the height of the obstacle, or the steepness of the climb, we now have proven that we will persist.

Address correspondence to: Hannah R. Turbeville, University of Michigan, 1550 East Medical Center Drive, Ann Arbor, Michigan 48109, USA. Email: Hannah.turbeville@physicianscientists.org.

\footnotetext{
1. Epstein RM, Krasner MS. Physician resilience: what it means, why it matters, and how to promote it. Acad Med. 2013;88(3):301-303.
} 